\title{
Evaluation of Two Internalizing Carcinoembryonic Antigen Reporter Genes for Molecular Imaging
}

\author{
Bhaswati Barat, Vania E. Kenanova, Tove Olafsen, Anna M. Wu \\ Crump Institute for Molecular Imaging, Department of Molecular and Medical Pharmacology, David Geffen School of Medicine at UCLA, \\ Box 951770, 570 Westwood Plaza, Los Angeles, CA 90095-1770, USA
}

\begin{abstract}
Purpose: The objective of this article is to develop internalizing positron emission tomography (PET) reporter genes for tracking genetically modified T cells in vivo.

Procedures: The transmembrane and cytoplasmic domains of the human transferrin receptor (TfR) and CD5 were each fused to the carcinoembryonic (CEA) minigene N-A3 and expressed in Jurkat $T$ cells. Internalization was evaluated by confocal microscopy or by intracellular uptake of ${ }^{125}$-labeled anti-CEA scFv-Fc. Reporter gene-transfected Jurkat xenografts in mice were analyzed by immunohistochemistry (IHC) and imaged by PET using ${ }^{124} \mathrm{I}-$ or ${ }^{64} \mathrm{Cu}-\mathrm{scFv}-\mathrm{Fc}$ as tracers.

Results: Surface expression of TR(1-99)-NA3 was lower than that of NA3-CD5. Both reporter genes were internalized following binding of the anti-CEA antibody fragment. IHC of tumors showed strong staining of NA3-CD5, whereas TR(1-99)-NA3 stained weakly. Specific targeting of TR(1-99)-NA3 or NA3-CD5 was shown by PET in xenografted mice.

Conclusions: The in vivo imaging studies suggest a potential application of the internalizing form of CEA (N-A3) as a PET reporter gene.
\end{abstract}

Key words: Antibody fragment, CEA reporter gene, Internalization, Jurkat, PET

\section{Introduction}

$\mathrm{T}$ he use of reporter genes enables researchers to investigate and monitor the expression of genes of interest both in cell culture and in vivo. Currently, in vivo imaging of gene transfer using reporter genes has become increasingly important in gene therapy trials due to the ability to noninvasively determine the level of gene transfer over time. In support of this, several groups have utilized optical,

Category and significance: An original article describing the design, generation, expression, and both in vitro and in vivo evaluation of two versions of an internalizing reporter gene. The significance of this approach lies in utilizing reporter protein internalization as a means of accumulation of the tracer in the target cells.

Correspondence to: Anna M. Wu; e-mail: awu@mednet.ucla.edu magnetic, or nuclear imaging techniques to evaluate gene transfer in animal models. In optical imaging studies, genes inducing fluorescence or bioluminescence have been used very successfully in mouse models $[1,2]$. However, these approaches are limited for human studies due to low tissue penetration and high scattering of the fluorescent or bioluminescent signal, and potential immunogenicity of the fluorescent or luminescent proteins. In contrast, positron emission tomography (PET) imaging of reporter gene expression is especially promising due to the inherent sensitivity, high resolution, and quantitation provided by PET, as well as the potential for clinical translation. In general, PET imaging techniques have used either enzymes or receptors as reporter genes. Established PET reporter systems include the herpes simplex virus 1 (HSV-1) thymidine kinase (tk; wild-type or sr39 mutant versions), 
using $\left[{ }^{18} \mathrm{~F}\right]$ or $\left[{ }^{124} \mathrm{I}\right]$-labeled nucleoside analogs, such as FPCV, FHBG, FHPG, and FIAU as reporter probes [3, 4]. Alternatively, the human dopamine type 2 receptor $\left(D_{2} R\right)$ and the somatostatin receptor (SSTr) in conjunction with their respective reporter probes, $\left[{ }^{18} \mathrm{~F}\right]$ FESP and $\left[{ }^{111} \mathrm{In}\right]-$ DTPA-D-Phe1-octreotide, have been extensively explored $[5,6]$. Additional reporter systems include the sodium iodide symporter (NIS) [7] and the human norepinephrine transporter (hNET), which has been recently used to image adoptively transferred human antigen-specific $\mathrm{T}$ cells in mice [8]. Each system has its advantages and limitations pertaining to immunogenicity and potential use as a suicide gene (tk), target specificity, uptake levels, background in normal tissues (NIS), and potential for unwanted biological activity $\left(\mathrm{D}_{2} \mathrm{R}, \mathrm{SSTr}\right.$, NIS, hNET) discussed in reference [9].

To reduce the likelihood of immunogenicity and/or ectopic expression, which is a concern for reporter gene expression, we designed a recombinant reporter gene based on the human carcinoembryonic antigen (CEA). CEA is an oncofetal antigen with a low level of expression in the lumen of the colon in normal human adults and no confirmed function [10]. CEA expression increases during the development of cancer, with $95 \%$ of human colon cancers being CEA positive, as well as a variable proportion of other carcinomas such as breast, lung, ovarian, and others [11]. In this work, the CEA-based reporter gene is detected by molecular imaging using a well-established radiolabeled anti-CEA antibody fragment as a reporter probe [12]. We have previously generated several engineered antibody fragments derived from the murine anti-CEA T84.66 monoclonal antibody $(\mathrm{mAb})[13]$. These recombinant antibody fragments have achieved high levels of uptake in tumor xenografts $(10-25 \% \mathrm{ID} / \mathrm{g})$ resulting in high contrast images following radiolabeling with the positron-emitting radionuclides ${ }^{64} \mathrm{Cu}$ or ${ }^{124} \mathrm{I}$ [14-16]. Expression of the fulllength, native human CEA in tumor cells has previously been used for the purpose of enhancing localization of radioiodinated intact mouse anti-CEA mAb [17]. In this study, significant amounts of radioactivity remained in the circulation 5 days post tracer injection, resulting in a low tumor-to-blood ratio $(0.9 \pm 0.6)$. This low ratio is probably attributed to the extended serum half-life of the intact mAb. We have recently reported on a noninternalizing CEA reporter gene that was evaluated by PET imaging [9]. This reporter gene was composed of the two CEA domains (N and A3) fused to a truncated version of the noninternalizing human Fc $\gamma$ RIIb cell surface receptor for stable membrane anchorage. Xenografts from NA3-Fc $\gamma$ RIIb transfected Jurkat T lymphocytes were visualized by PET imaging, using ${ }^{124}$ I-labeled anti-CEA single-chain (sc) Fv-Fc antibody fragment. In this study, a tumor-to-soft tissue ratio of 6.1 to 1 at $20 \mathrm{~h}$ was achieved.

Here we have investigated two internalizing versions of CEA (N-A3) as potential human reporter genes. The A3 domain contains the epitope recognized by the cT84.66 $\mathrm{mAb}$ [18]. The N-A3 minigene was fused to the human transferrin (CD71) receptor (TR(1-99)-NA3) and to the CD5 receptor
(NA3-CD5) replacing their extracellular domains (ECDs) while retaining the intracellular and transmembrane domains. Both constructs were transfected into Jurkat $\mathrm{T}$ cells, and the CEA reporter gene expression and ability to internalize were evaluated. We demonstrated that the expression of both versions of the internalizing CEA reporter gene was maintained in vivo, in tumor-bearing mice. An in vivo micro-PET imaging study using an ${ }^{124}$ I-labeled anti-CEA scFv-Fc antibody fragment as a probe showed specific localization to transfected Jurkat $\mathrm{T}$ cell xenografts. Thus, we have demonstrated the ability to generate and express an internalizing reporter gene on the surface of $\mathrm{T}$ cells, both in vitro and in vivo supporting the potential use of the internalizing forms of CEA as PET reporter genes.

\section{Materials and Methods}

\section{Generation of Recombinant CEA Reporter Gene}

The generation and expression of N-A3 as a fusion protein in a mammalian system has previously been described [9]. Here, the NA3 minigene was fused to the human transferrin receptor (TfR) (CD71) to produce a chimera between the N-terminal cytoplasmic and transmembrane sequences (1-99 amino acids) and the N-A3 gene of CEA (Fig. 1a). The TfR, lacking its ECD, was isolated from the pOTB7 vector (American Type Culture Collection [ATCC] MGC-3151, Manassas, VA) by polymerase chain reaction (PCR), using CD71 gene-specific primers. The PCR product was inserted into the pUC19 vector (New England Biolabs, Inc., Ipswich, MA) already containing the N-A3 minigene. The resulting full-length TR(1-99)-NA3 cDNA was then cloned into the mammalian expression vector pcDNA3.1 $1 \mathrm{TM} / \mathrm{neo}(-)$ (Invitrogen, Carlsbad, CA) on XbaI and EcoRI sites (Fig. 1a).

The human $\mathrm{CD} 5$ receptor gene, lacking its $\mathrm{ECD}$, was isolated from the pCMV-SPORT6 vector (ATCC MGC-34370) by PCR, using CD5 gene-specific primers. Site-specific mutations (T410A and T412A) were introduced in the cytoplasmic domain of CD5 using a QuickChange Site Directed Mutagenesis kit (Stratagene, La Jolla, CA) according to manufacturer's directions (Fig. 1b). The plus strand mutagenesis primers were T410A: 5'-CCATCGCAACCACGCGG CAACCGTCCG-3' and T412A: 5'-CGCAACCACGCGG CAGCCGTCCGATCCCATGC-3'. In order to generate the T410A/ T412A double mutant, CD5 with the introduced T410A mutation served as a DNA template for the second mutagenesis reaction with the T412A primer. The CD5 T410A/T412A was inserted into the pcDNA 3.1 $1^{\mathrm{TM}} / \mathrm{Zeo}(-)$ vector (Invitrogen), already containing the $\mathrm{N}$ A3 minigene on XhoI and EcoRI sites (Fig. 1b) [9].

\section{Cell Culture and Establishment of Stably Transfected Clones}

Human Jurkat cells ( $\mathrm{T}$ cell leukemia, ATCC TIB-152) were maintained as recommended. For stable transfection, $1 \times 10^{6}$ Jurkat cells were electroporated as described [19] with $10 \mu \mathrm{g}$ of linearized DNA (digested with $P v u$ I restriction enzyme). Clones expressing 
a
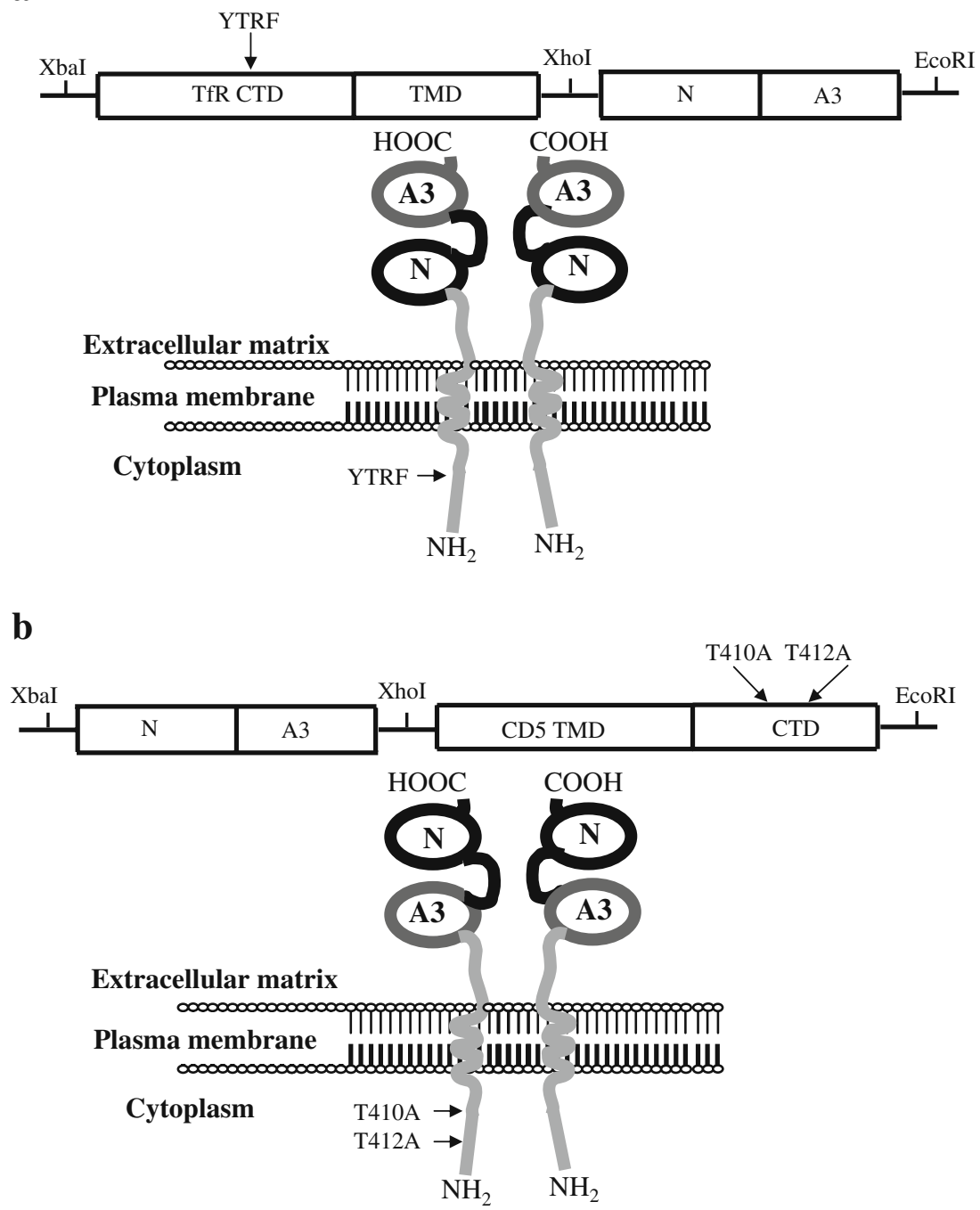

Fig. 1. Schematic gene and protein representations of the two internalizing recombinant CEA reporter molecules. TMD = transmembrane domain, CTD = cytoplasmic domain. The restriction enzyme cut sites are included. a TR(1-99)-NA3 reporter gene construct. The tetrapeptide sequence YTRF in the CTD of the TfR is responsible for internalization. $\mathbf{b}$ NA3-CD5 reporter gene construct. The two residues (T410 and T412) in the CTD of CD5 were mutated to alanine residues in order to eliminate normal biological activity.

TR(1-99)-NA3 were selected at $400 \mu \mathrm{g} / \mathrm{mL}$ of G418 (Calbiochem, San Diego, CA), while clones expressing NA3-CD5 were selected at $200 \mu \mathrm{g} / \mathrm{mL}$ of Zeocin (InvivoGen, San Diego, CA). Stably transfected cell lines were expanded and maintained in the respective drugs used for selection.

\section{Evaluation of Reporter Gene Expression by Flow Cytometry}

Jurkat cells (transfected and nontransfected) resuspended in $1 \times$ phosphate-buffered saline (PBS) containing 1\% fetal bovine serum (FBS) were incubated with $2 \mu \mathrm{g}$ of anti-CEA scFv-Fc H310A antibody fragment [12] for $1 \mathrm{~h}$ at $4^{\circ} \mathrm{C}$. Reporter protein expression was detected with $R$-phycoerythrin (PE)-conjugated goat antihuman (Fc $\gamma$-specific) mAb (Jackson Immunoresearch Laboratories, West Grove, PA). Antibody binding to the cells was analyzed by a FACSCalibur flow cytometer (Becton Dickinson, UK), detecting
PE (FL2, $\lambda_{\mathrm{em}}: 575 \pm 13 \mathrm{~nm}$ ) fluorescence (mean fluorescence intensity $[\mathrm{MFI}])$. All data were analyzed using the Cell Quest software (Becton Dickinson).

\section{Confirmation of Reporter Gene Internalization by Confocal Microscopy}

TR(1-99)-NA3 transfected and nontransfected Jurkat cells were plated on poly-L-lysine-coated glass coverslips (BD Biosciences, San Jose, CA) in 12-well plates, containing Cellgro RPMI 1640 Medium 1× (Mediatech Inc., Manassas, VA) supplemented with $5 \%$ FBS. One day after plating, cells were incubated with $2 \mu \mathrm{g}$ of cT84.66 anti-CEA antibody for $30 \mathrm{~min}$ at $4^{\circ} \mathrm{C}$ and then shifted to $37^{\circ} \mathrm{C}$. Internalization was assayed at 0 and $24 \mathrm{~h}$. At the designated time points $(0$ or $24 \mathrm{~h})$, cells were washed with PBS and fixed in $4 \%$ paraformaldehyde for $10 \mathrm{~min}$ at room temperature. To determine the expression of total recombinant reporter protein, 
following fixation cells were permeabilized with $0.2 \%$ Triton $\mathrm{X}$ 100 and blocked for 20 min with PBS containing 1\% FBS. Finally, cells were stained with Alexa Fluor 488-conjugated goat antihuman $\operatorname{IgG}(\mathrm{H}+\mathrm{L})$ antibody (Sigma-Aldrich) in PBS containing $1 \%$ FBS and $0.1 \%$ Triton X-100. The coverslips were mounted on glass slides and visualized under Leica TCS-SP inverted confocal microscope (Leica TCS-SP, Heidelberg, Germany) using a $\times 100$ oil immersion objective lens.

\section{Antibody Internalization Assay}

The anti-CEA cT84.66 antibody was labeled with ${ }^{125} \mathrm{I}$, using carrier-free $\left[{ }^{125} \mathrm{I}\right]-\mathrm{NaI}$ (MP Biomedicals, Inc., Irvine, CA) and Iodogen-coated tubes (Pierce, Rockford, IL), according to manufacturer's instructions. Unbound radioiodine was separated from the radiolabeled antibody using a PD-10 desalting column (GE Healthcare, Piscataway, NJ). NA3-CD5 transfected Jurkat cells were seeded in a 12-well plate at a density of $10^{6}$ cells $/ \mathrm{mL}$. Twenty-four hours later, cells were incubated in triplicate with $10^{5} \mathrm{cpm}$ of ${ }^{125}$ I-labeled cT84.66 antibody fragment in serum-free RPMI media on ice for $30 \mathrm{~min}$. The cells were then transferred to microfuge tubes, spun down, and washed three times with ice-cold RPMI media, followed by incubation at $37^{\circ} \mathrm{C}$ for $0,2,10,30$, and $60 \mathrm{~min}$ in RPMI media. After the indicated times, the medium was removed and cells were washed twice with $500 \mu \mathrm{L}$ of ice-cold glycine buffer (500 mM NaCl, $0.1 \mathrm{M}$ glycine, $\mathrm{pH} 2.5$ ) for $3 \mathrm{~min}$ in order to remove surface-bound radioiodinated antibody. The radioactivity that remained cell associated (intracellular) and in the glycine wash (surface-bound) was counted in a gamma counter (Wallac Wizard 3" 1480; Perkin Elmer Life and Analytical Sciences, Shelton, CT). The proportion of the internalized antibody was determined as the ratio of cell-associated radioactivity (internalized antibody) to the sum of cell-associated (internalized antibody) and acid-rinsed (surface-bound antibody) radioactivity.

\section{Establishment of Tumor Xenografts}

All animal studies were conducted under protocols approved by the Chancellor's Animal Research Committee at University of California, Los Angeles (UCLA). Reporter gene transfected and nontransfected Jurkat xenografts were established as described [9]. Briefly, for each study, three or four 7- to 8-week-old female athymic nude mice (Charles River Laboratories, Wilmington, MA) were sublethally irradiated with $450 \mathrm{rad}$ in a ${ }^{137} \mathrm{Cs}$ source irradiator (Mark-1, Model 68A; JL Shepherd and Associates, San Fernando, CA). Three days later, the irradiated mice were injected subcutaneously (s.c.) in the left shoulder region with a $1 / 1$ mixture of $1 \times 10^{6} \mathrm{~N}-\mathrm{A} 3$ transfected Jurkat and $1 \times 10^{6}$ irradiated (6500 rad) HT-1080 feeder cells (human fibrosarcoma, ATCC CCL-121). The right shoulder area was injected with the nontransfected Jurkat/feeder cell suspension as a negative control. Tumor masses were allowed to develop for about 20 days.

\section{Immunohistochemistry}

Immunohistochemical (IHC) staining was performed on both paraffin-embedded and frozen sections of transfected and nontransfected Jurkat cell tumors as described [9, 20]. TR(1-99)-NA3 transfected Jurkat tumor sections were stained with the intact
cT84.66 mAb, while the NA3-CD5 transfected Jurkat tumor sections were stained with the anti-CEA scFv-Fc H310A antibody fragment. Staining was developed using biotinylated goat antihuman $(\mathrm{H}+\mathrm{L})$ antibody $(1: 400)$ and horseradish peroxidaseconjugated avidin provided by the Vectastain Elite $\mathrm{ABC}$ kit (Vector Labs, Burlingame, CA). Stained tumor sections were examined at a magnification of $\times 20$ and photographed.

\section{${ }^{124}$ I Labeling of $s c F v-F c$ Antibody Fragments}

Radioiodination of the anti-CEA scFv-Fc fragments with ${ }^{124}$ I was performed by the Iodogen method as described [12]. Briefly, $0.2 \mathrm{mg}$ of antibody was incubated with $0.4-0.8 \mathrm{mCi} \mathrm{Na}^{124}$ I (IBA Molecular, Sterling, VA) using polypropylene tubes coated with $20 \mu \mathrm{g}$ Iodogen (Pierce). The labeling efficiencies were $81.5 \%$ for scFv-Fc H310A/H435Q and $89.9 \%$ for scFv-Fc H310A, as measured by instant TLC (Tec-Control kit; Biodex Medical Systems, Shirley, NY). Immunoreactivity was $63 \%$ for $\mathrm{scFv}-\mathrm{Fc}$ $\mathrm{H} 310 \mathrm{~A} / \mathrm{H} 435 \mathrm{Q}$ as determined by incubation of the labeled protein with excess LS174T cells (ATCC CL-188) overexpressing CEA.

\section{DOTA-Conjugation and ${ }^{64} \mathrm{Cu}$-Labeling of $s c F v-F c$ H310A Antibody Fragment}

Purified anti-CEA scFv-Fc H310A fragment (1 mg) was conjugated to $1,4,7,10$-tetraazacyclododecane- $N, N^{\prime}, N^{\prime \prime}, N^{\prime \prime \prime}$-tetraacetic acid (DOTA; Macrocyclics, Dallas, TX), using the water-soluble $\mathrm{N}$ hydroxysuccinimide method, as described [21, 22]. Following conjugation, the protein was extensively dialyzed in $0.9 \% \mathrm{NaCl}$ (pH 7.2) and concentrated.

The positron-emitting isotope ${ }^{64} \mathrm{Cu}$ (copper chloride in $0.1 \mathrm{~mol} / \mathrm{L}$ $\mathrm{HCl}$; radionuclide purity, $>99 \%$ ) was provided by Mallinckrodt Institute of Radiology (Washington University School of Medicine, St. Louis, WA). The DOTA-conjugated scFv-Fc H310A fragment $(0.3 \mathrm{mg})$ was incubated with $0.440 \mathrm{mCi}$ of ${ }^{64} \mathrm{Cu}$, as described [23]. The labeling efficiency was $56.2 \%$ as determined by instant TLC (Tec-Control kit, Biodex Medical Systems).

\section{Micro-PET Imaging and Statistical Analysis}

Mice were imaged using the micro-PET Focus 220 instrument (Siemens Preclinical Solutions, Inc., Knoxville, TN). Prior to ${ }^{124} \mathrm{I}$ imaging, thyroid and stomach uptake of radioiodine was blocked as described [15]. Three mice carrying Jurkat TR(1-99)-NA3 xenografts were injected in the tail vein with $139-143 \mu \mathrm{Ci}^{124}$ I-labeled scFv-Fc H310A/H435Q and serially imaged after 24 and $48 \mathrm{~h}$ of uptake time. Four mice carrying NA3-CD5 transfected Jurkat tumors were injected with $105-113 \mu \mathrm{Ci}^{124} \mathrm{I}$-labeled scFv-Fc H310A, and another four mice were injected with $99-102 \mu \mathrm{Ci}{ }^{64} \mathrm{Cu}$-labeled scFv-Fc H310A and serially imaged at 4 and $19-21 \mathrm{~h}$ post injection. Acquisition time was $10 \mathrm{~min}$ (one bed position), while mice were anesthetized with $2 \%$ isoflurane. Immediately after the last scan, anesthetized mice were euthanized by administration of $100 \mu \mathrm{l}$ of pentobarbital $(50 \mathrm{mg} / \mathrm{mL})$ intraperitoneally. Mice were dissected and blood, tumors, liver, spleen, kidneys, and lungs were harvested, weighted, and counted in a well counter (Cobra II Auto-Gamma; Packard, Illinois), and the $\% \mathrm{ID} / \mathrm{g}$ was calculated. The images were reconstructed using a filtered backprojection algorithm (FBP) [24]. All images were 
displayed by the AMIDE software [25], and regions of interest (ROI) were drawn as described [15]. The variance among mice is represented as mean $( \pm \mathrm{SD})$. The uptakes in blood, tumors, and organs $(\% \mathrm{ID} / \mathrm{g})$ were compared for significant difference using an unpaired Student $t$ test. A 1-tailed $P$ value of less than or equal to 0.05 was considered statistically significant.

\section{Results}

\section{Assembly and Expression of Internalizing Recombinant CEA Reporter Genes}

In order to create CEA as a recombinant reporter capable of internalization, the truncated form of CEA, N-A3, composed of 726 nucleotides [18], was genetically linked to one of two internalizing receptors, transferrin and CD5. The human TfR is a type II transmembrane protein (i.e., N-terminus inside, C-terminus outside) that is rapidly internalized upon binding with transferrin. The uptake and recycling of the TfR have been determined to be dependent on the amino acid motif Tyr-Thr-Arg-Phe (YTRF), which is located in the cytoplasmic domain (position 20-23 amino acid) [26]. In the present study, N-A3 was fused to the C-terminus (1-99 amino acids) of the intracellular and transmembrane regions of the TfR, replacing the extracellular transferrin-binding domain (Fig 1a). The construct was termed TR(1-99)-NA3.

The N-A3 minigene was also fused to CD5, which is a type I integral membrane protein and a key regulator of antigen receptor-mediated differentiation of $\mathrm{T}$ and $\mathrm{B}$ cells [27]. CD5 is composed of three extracellular cysteine-rich domains, a transmembrane region, and a cytoplasmic "immunoreceptor tyrosine-based activation motif" (ITAM) domain. Tyrosine 429 , located in the cytoplasmic domain of $\mathrm{CD} 5$, is responsible for internalization [27]. The N-A3 minigene was fused to the N-terminal side of the CD5 transmembrane and intracellular regions, replacing the extracellular cysteine-rich domains. The construct was termed NA3-CD5 (Fig 1b). Two conserved threonine residues (T410 and $\mathrm{T} 412$ ), located in the membrane proximal cytoplasmic region of CD5 and responsible for cellular signaling, were mutated to alanines for elimination of their biological activity [28].

In order to evaluate the expression of recombinant CEA reporters on Jurkat cells, TR(1-99)-NA3 and NA3-CD5 transfected Jurkat cells were incubated with the anti-CEA antibody and examined by flow cytometry. Nontransfected Jurkat cells were used as a negative control. Although the fluorescence intensity shifted both cell types to the right (Fig. 2), the MFI values differed almost four fold. The MFI value for TR(1-99)-NA3 transfected cells was 26 versus 92 for NA3-CD5 transfected cells. When cells were passaged and examined for expression over time, we noted that the cell surface expression of NA3-CD5 remained relatively stable, whereas TR(1-99)-NA3 expression of recombinant protein consistently dropped over time. Western blot analyses of the recombinant proteins (TR(1-99)-NA3 and
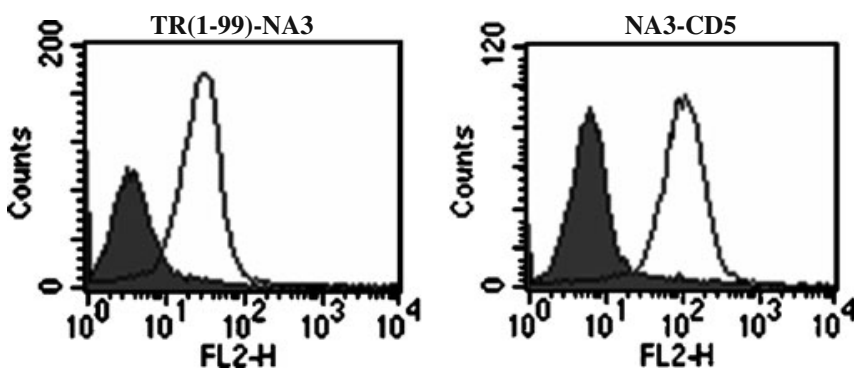

Fig. 2. Flow cytometry analysis of recombinant CEA reporter protein expression in Jurkat T cells. Nontransfected Jurkat (solid gray) and transfected Jurkat (black line).

NA3-CD5) showed that they migrated consistent with their predicted molecular weights (approximately $42 \mathrm{kDa}$ for both) under reducing conditions (data not shown).

\section{Internalization of Anti-CEA Antibody Fragments}

Internalization of the recombinant TR(1-99)-NA3 reporter protein, containing the TfR internalization sequence YTRF, was monitored by confocal laser scanning microscopy. Immunofluorescence, utilizing anti-CEA antibodies, showed surface staining of TR(1-99)-NA3 transfected Jurkat cells at $0 \mathrm{~h}$ and cytoplasmic staining at $24 \mathrm{~h}$ (Fig. 3a), which demonstrates internalization of the recombinant reporter protein. Control, nontransfected Jurkat cells showed no membrane staining at $24 \mathrm{~h}$ (Fig 3a).

The internalization of NA3-CD5 transfected Jurkat cells was investigated in the presence of ${ }^{125}$ I-labeled anti-CEA scFv-Fc H310A antibody fragment. The kinetics of NA3CD5 internalization showed that endocytosis was very rapid (Fig. 3b). Within $5 \mathrm{~min}$, more than $90 \%$ of the radiolabeled antibody fragment was internalized. The rapid internalization via NA3-CD5 was also observed by flow cytometry internalization studies (data not shown).

\section{Expression of Recombinant CEA Reporter Proteins in Transfected Jurkat Tumors}

Subcutaneous tumors were established in athymic nude mice by coinjecting transfected or nontransfected Jurkat cells with irradiated HT1080 human sarcoma feeder cells, respectively. Tumors were excised, frozen, and analyzed for expression of CEA by IHC. Immunohistochemical staining of tumors produced by TR(1-99)-NA3 transfected Jurkat cells showed that the expression of protein was very low (Fig. 4a), whereas the expression of NA3-CD5 transfected Jurkat tumors was more prominent (Fig. 4b).

\section{Micro-PET Imaging of Jurkat Xenografts Expressing TR(1-99)-NA3}

Micro-PET imaging was employed to evaluate the in vivo targeting of anti-CEA antibody fragments to the CEA reporter protein-positive tumors. For this purpose, nude 

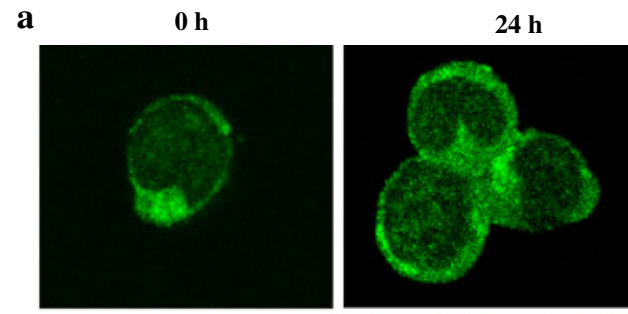

TR(1-99)-NA3

transfected

Jurkat

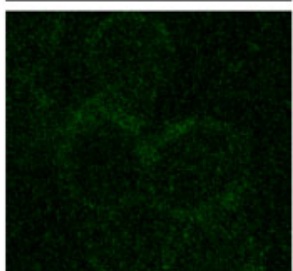

Non-transfected

Jurkat

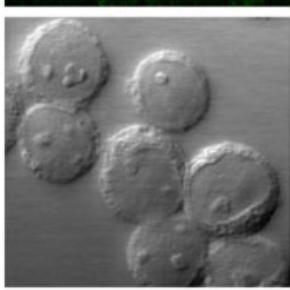

DIC image of Nontransfected Jurkat

b

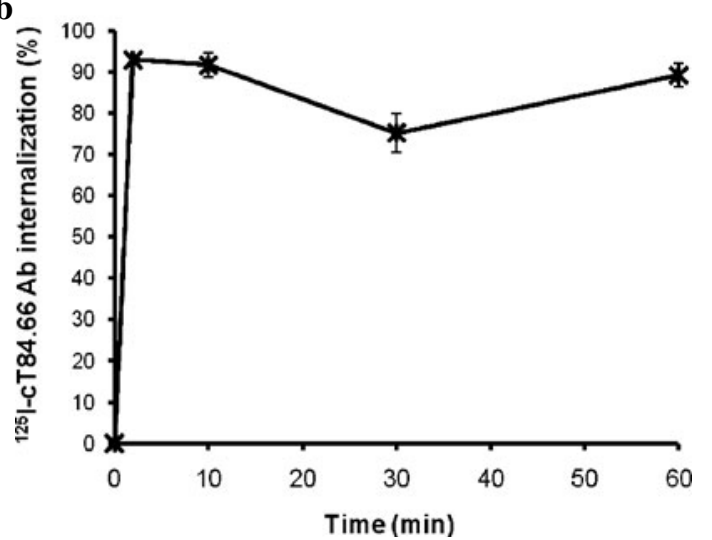

Fig. 3. Antibody-dependent internalization of recombinant CEA reporter protein. a Confocal microscopy image of TR(199)-NA3 transfected Jurkat cells labeled with anti-CEA cT84.66 mAb at 0 and $24 \mathrm{~h}$. Nontransfected Jurkat cells were used as a negative control, shown at $24 \mathrm{~h}$. A differential interference contrast (DIC) image of nontransfected Jurkat cells stained with cT84.66 at $24 \mathrm{~h}$ is shown. b Kinetics of NA3-CD5 internalization, determining uptake of anti-CEA cT84.66 in transfected Jurkat cells over time. Samples were assayed in triplicates and data are presented as mean \pm SEM.

mice ( $n=3)$ carrying TR(1-99)-NA3 (CEA-positive) and parental Jurkat (CEA-negative) tumors were injected with the ${ }^{124}$ I-labeled anti-CEA scFv-Fc H310A/H435Q antibody fragment. Whole-body micro-PET scans were obtained at 24 and $48 \mathrm{~h}$ post tracer injection. Uptake in the CEA-positive tumor and low activity in the CEA-negative control tumor were seen at $24 \mathrm{~h}$ (Fig. 5a). At 48 h, no signal was detected in the positive tumor. The average tumor-to-background ratio at $24 \mathrm{~h}$, based on ROI analyses, was 2.8. A biodistribution after the last scan $(48 \mathrm{~h})$ revealed that the TR(1-99)-NA3 expressing tumor had $0.95 \pm 0.007 \% \mathrm{ID} / \mathrm{g}$, which was significantly different $(P=.030)$ from the uptake in the negative tumor $(0.59 \pm 0.06 \% \mathrm{ID} / \mathrm{g})$, resulting in a positive to negative tumor ratio of 1.6. The radioactive uptake in the positive tumor was also significantly different from the activities in liver and kidneys $(P<.05)$, but not significantly different from the activities in spleen and lungs $(P>.05)$.

\section{Micro-PET Imaging of Jurkat Xenografts Expressing NA3-CD5}

Four mice bearing NA3-CD5 transfected Jurkat tumors were evaluated by micro-PET imaging using ${ }^{124}$ I-labeled scFv-Fc H310A fragment [12]. Although no signal was detected in the NA3-CD5-positive tumor in any of the images, biodistribution at $21 \mathrm{~h}$ showed uptake of $0.65 \pm 0.04 \% \mathrm{ID} / \mathrm{g}$ in the CEA-positive tumor and $0.30 \pm 0.14 \% \mathrm{ID} / \mathrm{g}$ in the control tumor, which was significantly different $(P=.023)$. Thus, the positive to negative tumor ratio was 2.2. The uptake in the positive tumor was not significantly different from the uptake in the kidneys $(P=.076)$ but was significantly higher than the uptakes in liver and spleen $(P<.05)$. Furthermore, there was significantly more radioactivity $(P<.05)$ in the blood $(2.1 \pm 0.31 \% \mathrm{ID} / \mathrm{g})$ and lungs $(1.2 \pm 0.38 \% \mathrm{ID} / \mathrm{g})$. Since the low uptake in NA3-CD5 xenografts may be due to rapid internalization upon tracer binding, another attempt to image NA3-CD5-positive tumors, using ${ }^{64} \mathrm{Cu}$-labeled anti-CEA scFv-Fc H310A fragment, was made with another group of four mice. In this study, the signal from the liver was very high $(22.5 \pm 4.48 \% \mathrm{ID} / \mathrm{g} ; P=.007)$, obscuring any signal coming from the tumor. In addition, radioactivities in spleen, kidneys, and lungs were also significantly higher $(P<.05)$ than the activity in the positive tumor at the time of euthanasia $(21 \mathrm{~h})$. Importantly, the radioactive uptake in the positive tumor $(2.78 \pm 0.84 \% \mathrm{ID} / \mathrm{g})$ was significantly more $(P=.040)$ than the uptake in the nontransfected, parental Jurkat tumor $(1.22 \pm 0.17 \% \mathrm{ID} / \mathrm{g})$, resulting in a positive to negative tumor ratio of 2.3 (Fig. 5b).

\section{Discussion}

The present study describes the construction and expression of two recombinant CEA reporter genes for the ultimate purpose of tracking genetically modified $\mathrm{T}$ cells in vivo. We have recently reported on a noninternalizing recombinant NA3-Fc $\gamma$ RIIb fusion reporter gene that was expressed in Jurkat cells [9]. Here, we proceeded to make internalizing reporter genes for the purpose of trapping and increasing the accumulation of radioactive tracer uptake in cells expressing these reporter genes. This would presumably translate into stronger signal in imaging applications. Two internalizing recombinant CEA reporter genes, TR(1-99)-NA3 and NA3CD5, were generated and stably transfected in human helper $\mathrm{T}$ Jurkat cells. The expression of the reporter proteins differed significantly when cells were evaluated by flow cytometry and when cells were grown as tumors and stained 

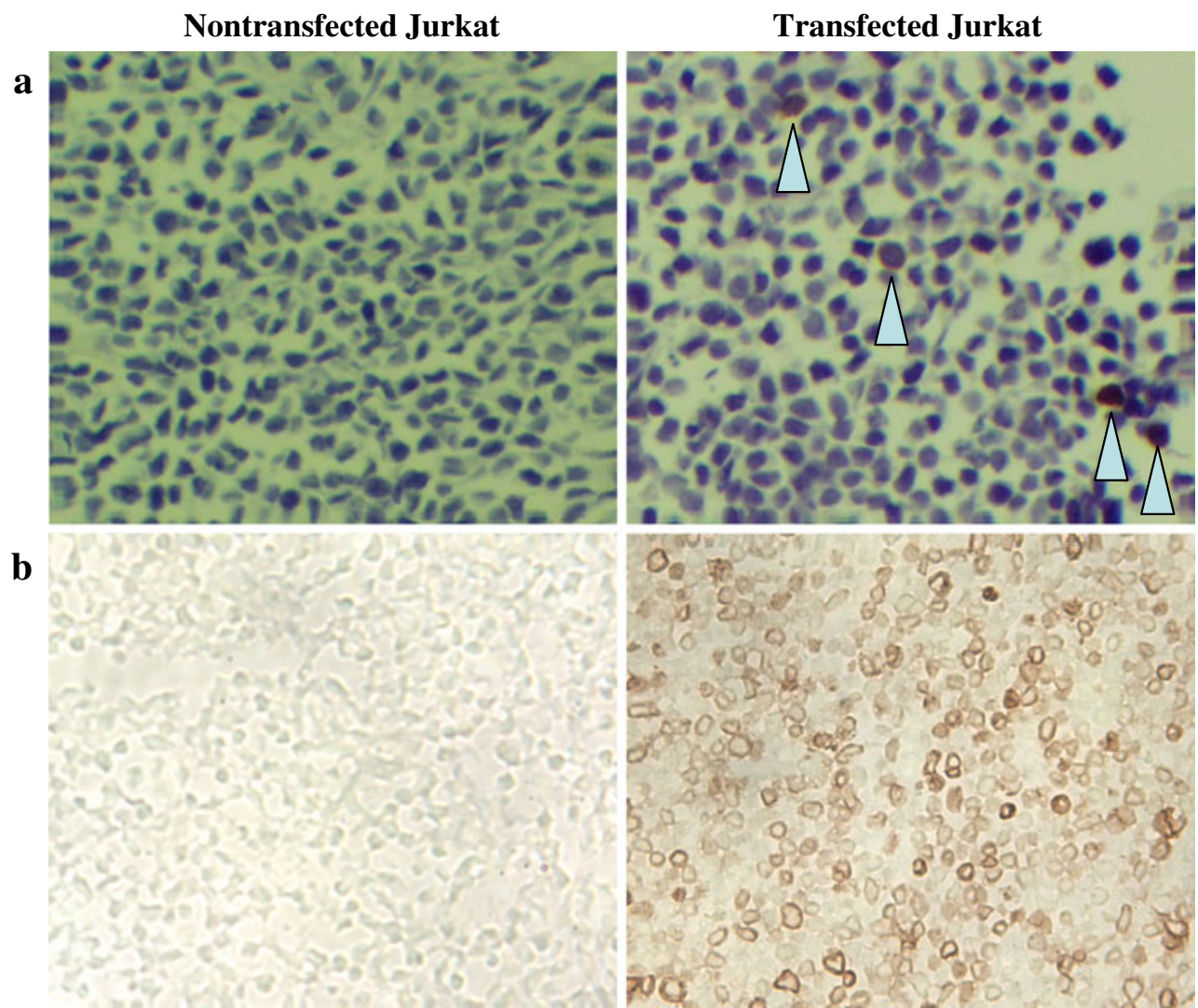

Fig. 4. Immunohistochemical staining of N-A3/CEA expression in tumors ( $\times 20$ magnification). a Paraffin-embedded sections of nontransfected and TR(1-99)-NA3 transfected Jurkat tumors stained with cT84.66 anti-CEA antibody. $\mathbf{b}$ Frozen sections of nontransfected and NA3-CD5 transfected Jurkat tumors stained with anti-CEA scFv-Fc H310A antibody fragment.

by IHC. The TR(1-99)-NA3 transfected Jurkat cells showed low levels of reporter protein expression, relatively to that of NA3-CD5. Nevertheless, specific targeting to TR(1-99)NA3 transfected Jurkat xenografts was demonstrated with the rapid clearing radioiodinated anti-CEA scFv-Fc H310A/ H435Q fragment $\left(t_{1 / 2 \beta}=6-8 \mathrm{~h}\right)[12,29]$ by in vivo microPET imaging. Reporter-positive tumors showed clear signal at $24 \mathrm{~h}$ (Fig. 5), and the tumor-to-background ratio, determined by ROI analysis of the images, was 2.8 . In our previous study with the noninternalizing NA3-Fc $\gamma$ RIIb reporter gene, we determined tumor-to-background ratio of 6.1 at $20 \mathrm{~h}$ in tumors, showing stronger IHC staining and expressing about 25,000 reporters per cell [9]. This observation suggests that even tumor masses with lower than 25,000 surface reporters (i.e., TR(1-99)-NA3 transfected Jurkat tumors) can still be detected by PET. Although higher surface expression was achieved with the NA3-CD5 reporter gene (result from flow cytometry analysis and IHC staining), micro-PET imaging using the slightly slower clearing scFv-Fc H310A antibody fragment $\left(t_{1 / 2 \beta}=21-\right.$ $27 \mathrm{~h})[12,29]$ did not improve the tumor uptake at $21 \mathrm{~h}$ with the positive to negative tumor ratio being 2.2. CD5 internalization efficiency has been found to be different with different antibody fragments [27]. Our in vitro internalization study, demonstrated rapid internalization of NA3CD5 recombinant reporters upon antibody binding. This could possibly explain the low uptake of radioiodinated antiCEA scFv-Fc fragment by NA3-CD5 expressing xenografts in the micro-PET imaging studies. Although radioiodination is simple and can be useful in preliminary imaging studies, internalized radioiodinated proteins are readily metabolized to iodotyrosines that are rapidly washed out of tissues and excreted into the urine in vivo [30]. Thus, direct radioiodination methods are generally not suitable for internalizing proteins as the signal is rapidly lost from the tissues. In contrast, indirect radiolabeling approaches, resulting in stable modification of the protein, are advantageous for internalizing proteins as the label is retained and accumulates in the cell over time. However, the disadvantage is that activity is also retained in tissues involved in the clearance of the protein (i.e., liver and kidneys), limiting imaging of tumors proximal to these organs. When NA3-CD5 transfected Jurkat xenografts were imaged with ${ }^{64} \mathrm{Cu}$-DOTA$\mathrm{scFv}-\mathrm{Fc} \mathrm{H} 310 \mathrm{~A}$, a higher radioactive uptake in the positive tumor was measured compared to the one achieved with the radioiodinated fragment. However, use of ${ }^{64} \mathrm{Cu}$ also resulted in high normal tissue activities, especially in the liver, which prevented visualization of the signal in the positive tumors. Although the present study shows that the surface expression level of NA3-CD5 is higher than that of TR(1-99)-NA3, the imaging study suggests that it is not high enough to enable sufficient accumulation in the tumors for production of high 


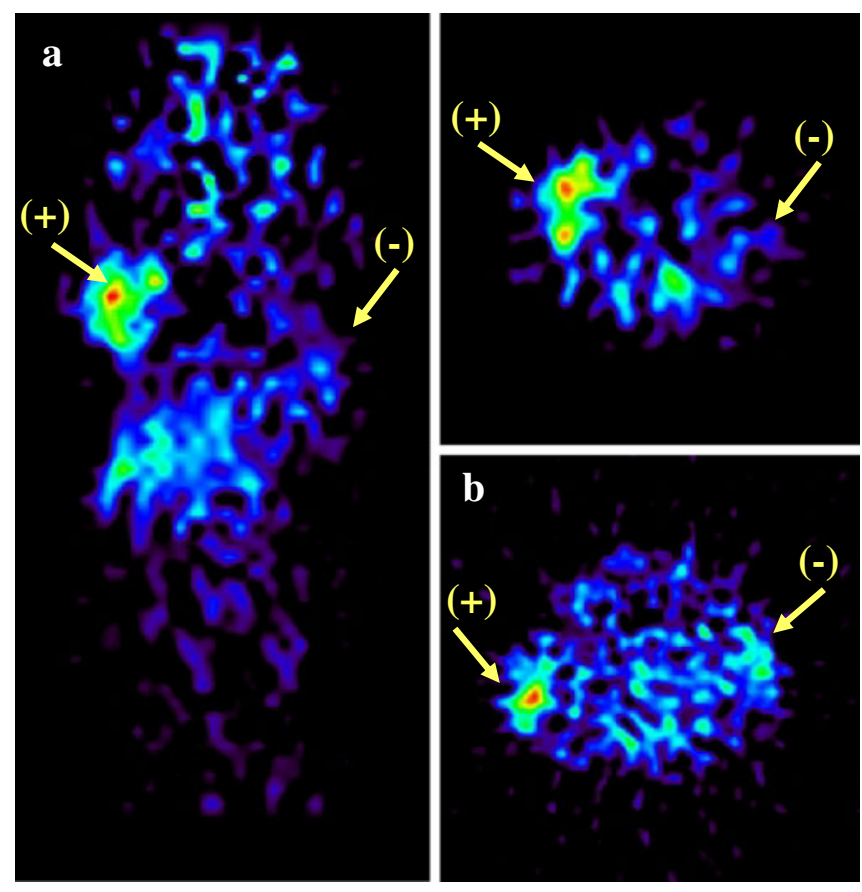

Fig. 5. Micro-PET imaging of CEA reporter proteins. a Coronal and transverse views of a nude mouse with TR(1-99)-NA3 transfected $(+)$ and nontransfected $(-)$ xenografts, injected with $140 \mu \mathrm{Ci}$ of ${ }^{124}$ I-labeled anti-CEA scFv-Fc H310/H435Q fragment at $24 \mathrm{~h}$ after injection. $\mathbf{b}$ Transverse view of a nude mouse with NA3-CD5 transfected (+) and nontransfected (-) xenografts, injected with $100 \mu \mathrm{Ci}$ of ${ }^{64} \mathrm{Cu}$-labeled anti-CEA scFv-Fc $\mathrm{H} 310 \mathrm{~A}$ fragment at $21 \mathrm{~h}$ postinjection.

contrast images. The signal in the liver can be eliminated by using a smaller antibody fragment that clears through the kidneys such as the diabody ( $55 \mathrm{kDa}, \mathrm{scFv}$ dimer) as a PET tracer. This fragment has demonstrated targeting to antigen-positive tumors, while clearing rapidly from the circulation, attaining favorable tumor-to-blood ratios at early times [15].

In our previous study with the noninternalizing reporter gene, cell populations with different expression levels (high, medium and low) were generated [9]. In this study, transfections of the Jurkat cells with the N-A3 reporter genes were inefficient. Only one NA3-CD5 expressing clone was found. This clone was a high expressor (MFI >95) that maintained its high expression over time. Transfection of TR (1-99)-NA3 reporter gene yielded several clones. However, despite multiple attempts, only a few clones with limited surface expression of TR(1-99)-NA3 reporter protein were obtained. Jurkat cells are inherently difficult to transfect. A study showed that electroporation of Jurkat cells resulted in $15.83 \pm 3.5 \%$ transfection efficiency [31]. For this reason, several protocols (different voltage and number of pulses) were developed to increase transfection efficiency. Since the transfection efficiency was not a problem with the noninternalizing NA3-Fc $\gamma$ RIIb [9], other transfection approaches than electroporation were not attempted. Another observation similar to our study showed that chimeric feline TfR formed by feline TfR cytoplasmic tail, transmembrane, and stalk sequences with antibody single-chain variable fragments $(\mathrm{scFv})$ derived from antiviral (canine parvovirus [CPV]) $\mathrm{mAb}$ was difficult to generate stably Jurkat cell lines expressing levels of chimeric receptors high enough for detection [32]. This experiment also supports the low transfection efficiency and low-level expression of chimeric receptors on Jurkat cells.

A potential problem that could affect expression of the TR (1-99)-NA3 reporter gene may be the reverse orientation of the N-A3 minigene (Fig. 1a), leading to incorrect folding and/or perturbed tertiary structure. The human TfR has been previously fused to the human IgG1 $\mathrm{Fc}$ region and expressed on the surface of baby hamster kidney (BHK) cells [33]. In this work, the $\mathrm{Fc}$ region was also inserted in the reverse orientation, and high surface expression of the chimera from pCDNA 3.1 $(-)$ vector following transfection was documented. Since CEA is a member of the immunoglobulin supergene family, it is unlikely that the reverse orientation of N-A3 affects its threedimensional structure. Another potential issue with expressing recombinant proteins in Jurkat cells may be the promoter used. The strength of various viral promoters (CMV, RSV, and SV40) was examined in Jurkat cells [34]. It was found that the CMV promoter was 10 -fold stronger than the RSV promoter and significantly stronger than the SV40 promoter in Jurkat cells. In this work, the pCDNA 3.1(-) vector that contains the CMV promoter for gene expression was used. Since the orientation of the gene and the promoter used do not seem to be an issue for expression in the work, the problem is probably the Jurkat cell line.

In conclusion, the construction and characterization of two recombinant internalizing CEA reporter genes with apparently different internalization rates were completed. The ability to noninvasively image reporter genes with PET was shown using mice bearing TR(1-99)-NA3 reporter gene transfected Jurkat xenografts. The strategy of using N-A3 as reporter gene is not limited to $\mathrm{T}$ cells only. These studies also suggest potential application of the recombinant CEA (N-A3 minigene) as a reporter for noninvasive imaging of any cell type (e.g., B cells, stem cells, or as a transgene in animals). Future studies could optimize this system through improvement of the recombinant reporter gene design (N-A3 orientation and internalization module) or through the use of PET reporter probe of a smaller size. Of the two versions of internalizing recombinant CEA reporter proteins made, NA3-CD5 was the faster internalizing molecule than TR (1-99)-NA3. Although we were not able to detect a signal from the NA3-CD5 reporter positive tumor by micro-PET imaging with the ${ }^{64} \mathrm{Cu}$-labeled anti-CEA scFv-Fc antibody fragment, the biodistribution results indicated specific targeting and accumulation of activity. Shifting to a smaller antibody fragment, like the anti-CEA diabody or minibody for the PET reporter probe, could improve image contrast 
and target detectability. Finally, the immunogenicity that both reporter genes may exhibit in humans needs to be formally evaluated.

\section{Conclusions}

In this study, two internalizing CEA reporter genes, TR (1-99)-NA3 and NA3-CD5, were expressed on the surface of Jurkat $\mathrm{T}$ cells. Expression was confirmed by flow cytometry and IHC. In serial small animal PET imaging studies, only the TR(1-99)-NA3 expressing Jurkat tumor xenografts could be visualized by using an ${ }^{124}$ I-labeled tracer. By using a ${ }^{64} \mathrm{Cu}$-labeled tracer, the NA3-CD5 reporter expressing Jurkat xenografts could be imaged. However, signal accumulation was low due to rapid internalization and low expression levels of the reporter genes on the cell surface. Optimization of the reporter gene design and boost in the expression level is required for improvement of the signal accumulation in the reporter gene expressing tumors.

Acknowledgments. This work was supported by the National Institute of Health (NIH) grants CA 043904 and CA 086306. A.M. Wu is a member of the UCLA Jonsson Comprehensive Cancer Center (NIH CA 016042). We thank the UCLA Brain Research Institute Carol Moss Spivak Cell Imaging Facility for providing confocal microscopy as well as the UCLA Jonsson Comprehensive Cancer Center and Center for AIDS Research Flow Cytometry Core Facility. We are especially grateful to Dr. David Stout, Waldemar Ladno, and Judy Edwards at UCLA for their assistance with the micro-PET scans. We thank Sofia Loera at the Anatomic Pathology Core Facility (City of Hope Comprehensive Cancer Center) as well as Lojze M Smid and Graham Cole at the Department of Molecular and Medical Pharmacology, UCLA for performing IHC.

Conflict of Interest. The authors declare that they have no conflict of interest.

Open Access. This article is distributed under the terms of the Creative Commons Attribution Noncommercial License which permits any noncommercial use, distribution, and reproduction in any medium, provided the original author(s) and source are credited.

\section{References}

1. Ray P, Gambhir SS (2007) Noninvasive imaging of molecular events with bioluminescent reporter genes in living subjects. Methods Mol Biol 411:131-144

2. Weissleder R, Ntziachristos V (2003) Shedding light onto live molecular targets. Nat Med 9:123-128

3. Gambhir SS, Bauer E, Black ME et al (2000) A mutant herpes simplex virus type 1 thymidine kinase reporter gene shows improved sensitivity for imaging reporter gene expression with positron emission tomography. Proc Natl Acad Sci USA 97:2785-2790

4. Tjuvajev JG, Doubrovin M, Akhurst T et al (2002) Comparison of radiolabeled nucleoside probes (FIAU, FHBG, and FHPG) for PET imaging of HSV1-tk gene expression. J Nucl Med 43:1072-1083

5. MacLaren DC, Gambhir SS, Satyamurthy N et al (1999) Repetitive, non-invasive imaging of the dopamine D2 receptor as a reporter gene in living animals. Gene Ther 6:785-791

6. Rogers BE, McLean SF, Kirkman RL et al (1999) In vivo localization of [(111)In]-DTPA-D-Phe1-octreotide to human ovarian tumor xenografts induced to express the somatostatin receptor subtype 2 using an adenoviral vector. Clin Cancer Res 5:383-393

7. Cho JY, Shen DH, Yang $\mathrm{W}$ et al (2002) In vivo imaging and radioiodine therapy following sodium iodide symporter gene transfer in animal model of intracerebral gliomas. Gene Ther 9:1139-1145
8. Doubrovin MM, Doubrovina ES, Zanzonico P, Sadelain M, Larson SM, O'Reilly RJ (2007) In vivo imaging and quantitation of adoptively transferred human antigen-specific $\mathrm{T}$ cells transduced to express a human norepinephrine transporter gene. Cancer Res 67:11959-11969

9. Kenanova V, Barat B, Olafsen T et al (2009) Recombinant carcinoembryonic antigen as a reporter gene for molecular imaging. Eur J Nucl Med Mol Imaging 36:104-114

10. Shively JE, Beatty JD (1985) CEA-related antigens: molecular biology and clinical significance. Crit Rev Oncol Hematol 2:355-399

11. Benchimol S, Fuks A, Jothy S, Beauchemin N, Shirota K, Stanners CP (1989) Carcinoembryonic antigen, a human tumor marker, functions as an intercellular adhesion molecule. Cell 57:327-334

12. Kenanova V, Olafsen T, Crow DM et al (2005) Tailoring the pharmacokinetics and positron emission tomography imaging properties of anti-carcinoembryonic antigen single-chain Fv-Fc antibody fragments. Cancer Res 65:622-631

13. Wu AM, Senter PD (2005) Arming antibodies: prospects and challenges for immunoconjugates. Nat Biotechnol 23:1137-1146

14. Olafsen T, Cheung CW, Yazaki PJ et al (2004) Covalent disulfidelinked anti-CEA diabody allows site-specific conjugation and radiolabeling for tumor targeting applications. Protein Eng Des Sel 17:21-27

15. Sundaresan G, Yazaki PJ, Shively JE et al (2003) 124I-labeled engineered anti-CEA minibodies and diabodies allow high-contrast, antigen-specific small-animal PET imaging of xenografts in athymic mice. J Nucl Med 44:1962-1969

16. Wu AM, Yazaki PJ, Tsai S et al (2000) High-resolution microPET imaging of carcinoembryonic antigen-positive xenografts by using a copper-64-labeled engineered antibody fragment. Proc Natl Acad Sci USA 97:8495-8500

17. Raben D, Buchsbaum DJ, Khazaeli MB et al (1996) Enhancement of radiolabeled antibody binding and tumor localization through adenoviral transduction of the human carcinoembryonic antigen gene. Gene Ther 3:567-580

18. You YH, Hefta LJ, Yazaki PJ, Wu AM, Shively JE (1998) Expression, purification, and characterization of a two domain carcinoembryonic antigen minigene (N-A3) in Pichia pastoris. The essential role of the Ndomain. Anticancer Res 18:3193-3201

19. Olafsen T, Kenanova VE, Wu AM (2006) Tunable pharmacokinetics: modifying the in vivo half-life of antibodies by directed mutagenesis of the Fc fragment. Nat Protoc 1:2048-2060

20. Olafsen T, Tan GJ, Cheung CW et al (2004) Characterization of engineered anti-p185HER-2 (scFv-CH3)2 antibody fragments (minibodies) for tumor targeting. Protein Eng Des Sel 17:315-323

21. Lewis MR, Kao JY, Anderson AL, Shively JE, Raubitschek A (2001) An improved method for conjugating monoclonal antibodies with $N$ hydroxysulfosuccinimidyl DOTA. Bioconjug Chem 12:320-324

22. Yazaki PJ, Wu AM, Tsai SW et al (2001) Tumor targeting of radiometal labeled anti-CEA recombinant T84.66 diabody and $\mathrm{t} 84.66$ minibody: comparison to radioiodinated fragments. Bioconjug Chem 12:220-228

23. Olafsen T, Kenanova VE, Sundaresan G et al (2005) Optimizing radiolabeled engineered anti-p185HER2 antibody fragments for in vivo imaging. Cancer Res 65:5907-5916

24. Defrise M, Kinahan PE, Townsend DW, Michel C, Sibomana M, Newport DF (1997) Exact and approximate rebinning algorithms for 3D PET data. IEEE Trans Med Imaging 16:145-158

25. Loening AM, Gambhir SS (2003) AMIDE: a free software tool for multimodality medical image analysis. Mol Imaging 2:131-137

26. Collawn JF, Lai A, Domingo D, Fitch M, Hatton S, Trowbridge IS (1993) YTRF is the conserved internalization signal of the transferrin receptor, and a second YTRF signal at position 31-34 enhances endocytosis. J Biol Chem 268:21686-21692

27. Lu X, Axtell RC, Collawn JF, Gibson A, Justement LB, Raman C (2002) AP2 adaptor complex-dependent internalization of CD5: differential regulation in T and B cells. J Immunol 168:5612-5620

28. Vila JM, Calvo J, Places L et al (2001) Role of two conserved cytoplasmic threonine residues (T410 and T412) in CD5 signaling. J Immunol 166:396-402

29. Kenanova V, Olafsen T, Williams LE et al (2007) Radioiodinated versus radiometal-labeled anti-carcinoembryonic antigen single-chain $\mathrm{Fv}-\mathrm{Fc}$ antibody fragments: optimal pharmacokinetics for therapy. Cancer Res 67:718-726 
30. Eary JF, Appelbaum FL, Durack L, Brown P (1989) Preliminary validation of the opposing view method for quantitative gamma camera imaging. Med Phys 16:382-387

31. Pepe J, Rincon M, Wu J (2004) Experimental comparison of sonoporation and electroporation in cell transfection applications. Acoust Res Lett Online 5:62-67

32. Hueffer K, Palermo LM, Parrish CR (2004) Parvovirus infection of cells by using variants of the feline transferrin receptor altering clathrin-mediated endocytosis, membrane domain localization, and capsid-binding domains. J Virol 78:5601-5611

33. Stabila PF, Wong SC, Kaplan FA, Tao W (1998) Cell surface expression of a human IgG Fc chimera activates macrophages through Fc receptors. Nat Biotechnol 16:1357-1360

34. Sutherland LC, Williams GT (1997) Viral promoter expression in CEM-C7 and Jurkat human T-lymphoid cell lines. J Immunol Methods 207:179-183 